\title{
Enhancement of technical value of oil palm (Elaeis guineensis Jacq.) waste trunk through modification with 1,3-dimethylol-4,5-dihydroxyethyleneurea (DMDHEU)
}

\begin{abstract}
Malaysia is the biggest producer of palm oil in the world. The production generates over 70 metric tons of waste trunks per hectare during replantation. Such an abundant feedstock should be considered a valuable raw material rather than an agro-waste. An approach for enhancement of low density trunks through the treatment with 1,3-dimethylol-4,5dihydroxyethyleneurea (DMDHEU) was investigated. The treatment resulted in a great improvement in the properties of the material: $45 \%$ density gain, water absorption and thickness swelling reduced by 48 and $43 \%$, respectively, 2.3-fold increase in hardness as well as 3.8-fold and 3.6-fold increase in bending strength and modulus of elasticity, respectively, were observed. Thus, enhancement in the physical and mechanical performance of the material as well as the increased aesthetic value due to the color changes proved the approach to be effective for the conversion of waste biomass to new products.
\end{abstract}

Keyword: Heat release rate; Furfuryl alcohol; Weight percentage gain; Laminate veneer lumber; Thickness swell 